\title{
Research on Visual Graphic Communication in New Media Context
}

\author{
Yun Li \\ Department of visual communication \\ Yantai Nanshan University \\ Longkou, Shandong, China
}

\begin{abstract}
One of the significant features of modern design is the graphics of visual image. As a visual symbol, graphics disseminates information through modern media and has become one of the basic elements of modern design. Its function is far beyond the traditional aesthetic form and has expanded to become a carrier of visual art. In the new media era, visual artistic form has gradually become an important language of communication. This paper will discuss how to use graphics to carry out effective information dissemination.
\end{abstract}

Keywords—new media; visual graphic communication

\section{INTRODUCTION}

Since twentieth Century, digital information technology has been the exchange, outdoor multi angle digital media exchange mode, multi platform digital media exchange way to make great changes in social environment. Economic development has led to the progress of the technology, the use of visual graphics mode of communication has become the main way to promote the development of digital information transmission mode and level in the current development of digital media technology. The so-called new media is a new technology to promote the continuous extension of the concept, it is generated in the computer information processing technology, relying on the Internet and digital technology, in order to restore people's daily life in a virtual way of information dissemination and exchange activities. The new media has the direct and the mass communication. It is a highly complex communication form. Its main form is the Internet, mobile phone, electronic games, digital interactive television, outdoor electronic, etc.. Compared to the newspapers, outdoor, radio, television, the traditional sense of the media, the new media is vividly called the fifth media". New media in the technology, communication, performance, visual language is more than in the past to change and breakthrough. Due to the influence of new media digital technology, visual communication design has been expanded, such as interactive entertainment design, network structure and interface design, multimedia design, application software and digital equipment display interface design.

The new media context is a media communication mode which will be linked with various media channels, digital information technology, outdoor multi angle digital media exchange mode, multi platform digital media information exchange. In the development process of the whole form, the main development condition of this kind of ideology, as a new factor of the construction of the new media, will be the final transmission mode and factor analysis.

With the continuous development of information, electronic information computing continue to show the way, with the establishment of electronic information platform, visual expression by traditional printing methods into expression by electronic technology, a large number of studies have shown that people convey information visual accounted for two thirds of the. Visual graphics is the main form of visual communication, so in the new media era of new technology, the emergence of new media era background, the visual graphics communication should be how to break it? This is the focus of our study.

\section{VISUAL GRAPHICS COMMUNICATION CHARACTERISTICS}

Traditional visual design is carried out in two dimensional space, whether it is text, graphics or color, it is the purpose of transmitting information through two dimensional space, because human understanding of things is a development process, only from the two-dimensional and three-dimensional vision is unable to complete the full understanding of a thing, while the four-dimensional space can guide people to receive the visual information, causing people's thinking, experience and aftertaste. The dissemination and reception of four dimensional information is the unique advantage of new media technology. Under the new media context, multi dimensional experience is possible, in the new media context, the visual communication designer how to improve the use of visual language is particularly important.

Graphics are different from the text, music, graphics is a non - text of the world language, is one of the main means of mass communication, graphics in the modern design is the biggest feature of the visual image is the purpose of the dissemination of information. As the basic elements of modern design, its function is far beyond the traditional aesthetic form, has been expanded into a kind of visual art as the carrier of the media, a kind of visual art as the form of communication language. 
Graphics and text is a kind of carrier tool for human communication, graphics are the other visual communication between characters and art. The biggest difference between graphics and text is that it is not restricted by the national region. Works of art with the distinction between art works focus on the artist's personal feelings of the outlet, can not consider the work of the value, and can be accepted by the public. The characteristics of graphics language not only to be able to convey information directly and accurately, to mobilize the psychological, more important is to take into account the understanding of the audience, the realization of the soul touching and communication, and finally reached an emotional exchange. As the saying goes, "a picture is worth a thousand words". Therefore, graphic language can attract attention and can keep the strong visual impact force.

\section{VISION AND MEDIA}

Visual communication to convey information for the purpose, the final performance of the interface communication is always also cannot do without the media, the relationship between the media and visual communication between the interactive media, which reflects the design, and makes the design limitations, design is subject to the media, and is one of the power generated by the new media, if no visual media, we will not experience the colorful nature and active in the inner emotions, like music experience cannot behave like in concept is rational in design, expression and appeal directly to the vision, the modern society is experiencing a change from culture to culture text graphics, visual communication in contemporary era, culture is becoming a kind of visual culture, people in order to express their feelings and will, often resort to various media as a carrier of emotions. From the perspective of information communication, the continuous evolution of the material and technical carrier of cultural communication, so that different periods of social and cultural characteristics of different media and communication effects.

Since the beginning of human history, that is, communication behavior, in the language before the birth of human gestures expression to convey, and then to a rope or use sounds to express thoughts. Printing has not yet been invented before, people in written communication, with the progress of the times, until the 19th century printing developed, by communication of point and line link expansion grounds extravagant extending communication activities, traffic tools of progress more propagation speed accelerate and expand the scope of, people then use newspapers, magazines, movies, radio, television, photography, video and other media, and further promote the effectiveness of the spread continues to expand, so-called "communication", some "between man and man, by symbols to convey information process. Communication involved in almost everything that human beings do, from the most simple oral communication to complex need treatment in line with the electronic information dissemination, almost all of the visual convey design forms are inseparable from many media, design behavior because of mass media and the repeated cycles to communicate information, selling goods, promotional services, communication intention. The information dissemination in the Internet age is the most important and far-reaching influence in the history of mankind. From the development of history, the media has experienced oral culture, written and print culture and electronic media three stages. In the mass media, the form of written culture is becoming more and more dependent on the electronic media, and the revolution of information communication has made it clear that the power of the network. In the past, books, newspapers, documents, letters, and other information transfer mode and material transmission are consistent, the emergence of new media to make the world a fundamental change in the face of".

Along with the progress of modern society and the development of science and technology, the communication activities have penetrated into every corner of life and the activities of human beings. Rely on visual communication information is on the trend, so as to the external life and inner spirit of the continuous penetration and influence, forming a comprehensive quality of the cultural phenomenon, how to obtain information, how to interact with each other's life space, common cultural factors, the formal intention to promote, so that the media's stick to come and go freely.

\section{Visual GRAPHICS IN NEW MEDIA}

In fact, the contemporary culture not only completed the shift to the visual culture, but also completed the extension to the network world. Compared with the traditional delay of communication of text, visual electronic media closer to realtime communication; with the help of modern information technology, visual culture gradually came into the era of digital virtual image, the undisputed, video, images and other visual information in the Internet communication and Sheng, has its inherent advantages.

First, Internet users exhibit a "reading impulse", this is not the source to people for the text of the fear, tired, or is a reactionary, but people are more willing to pursue an image changes and visual satisfaction. Image information is one of the important channels for people to get information. Dale (1969) of the "Tower of experience" points out that people get more than $70 \%$ of information through visual means, in the Internet age is no exception. According to USA scholars found that $79 \%$ of Internet users, reading the information on the Internet is to sweep the. Network communication is a kind of information which contains the text, image, video, voice and other kinds of symbols. From the perspective of people's information, graphics, images, audio, the main appeal to people's feelings, and the text, language, and the main appeal to people's sense of reason. For the former, it is only to mobilize the visual, auditory passive perception can be, the main influence of people's emotions; for the latter, we not only to mobilize the visual, auditory and other cognitive, more important is to mobilize the thinking to understand the reason. Obviously, for the main body, the former is much easier.

Secondly, the kind of color on the web page, pictures are very suitable for users to read the psychological and behavior, can greatly stimulate their interest and emotion. Digital than narrative creation is graphic language like confluence of 
technical text, here, literature easily by indirect image of "language like" turned into a visual image, the former "language arts" into the graphic compatible interface text, that through the pages of books written solutions to read and experience reduction to obtain the rich imagination of the indirect image, has let in teletext compatible, endowment tableaux, affectionately, interface transfer electronic fast food.

Again, symbolic image is symbolic image, be "portrait" in very close to the real things of visual language and its "refers to" and "signified" with a high degree of compliance and structure unlike language as burdensome syntax specification, image and its interpretation can be transferred directly to people's perceptual experience and perceptual thinking and is less subject to the limitations of the cultural level.

From the view of human civilization evolution history, the creation and development of the visual culture is the development of the visual media and communication. It not only makes the visual language culture from the simple, simple expression gradually to the tool, electronic, digital and multimedia integrated transport direction, but also from graphics, image to the image, static modeling to time and network interactive form, and in the dissemination of visual language, from the imitation, record to emotional, the idea of the overall trend and features. That is to say, the visual language of form has not only has the reproduction or objective image myriad changes, the function of human emotional catharsis, it can express abstract ideas through a combined structure grammar metaphor and symbol, and it is still in development in further together with medium of words, sounds and symbols in symbiotic complementary gradually into the comprehensive media language use. Film, animation and interactive new media is a typical phenomenon of the rise.

It can be said that the language form and the dissemination of the typical visual image features have been effectively to the foreground and center of the human civilization landscape, playing more and more important role in communication and cultural communication. This is known as the visual culture of the "image era" is more and more profound influence on the human political, economic and social life, and showing a new look and ecological, almost ubiquitous.

\section{How to EfFectively Use the Mass Media of VISUAL GRAPHICS}

From graphics, images to the image of the visual media technology, language form and expression of revolutionary development, leading to a comprehensive combination of economic, cultural, scientific and artistic features of the field of visual culture, in recent decades, from the traditional painting, technology, design, image and other areas rapidly expanded to include digital network, game entertainment and various types of interactive new media. Computer production, new printing technology and media materials, so that we have today's graphic communication and visual design more exquisite and moving; electronic image technology upgrade, digital media, network and the popularity of new interactive media forms a large number of sound, image, video, video, video games, interactive digital communication continues to introduce new and innovative. This trend continues to develop, regardless of its connotation, nature, or the size of a huge change, not only to bring a major adjustment of the cultural existence form, structure, but also to accelerate the diversification of information resources and commercialization, so that the traditional concept of culture, acceptance and the overall appearance of a qualitative change.

Since a new communication media and Industry under the background of the form of visual language beyond the traditional media, technology and applications has difficult to simply use the past habits of pictures, graphics, images, video etc. term to system description, is also difficult to purely semantic traceability of empirical method, to derive the so-called essence and scientific concept in the so-called image, graphic, picture external historical and cultural meaning and translation differences. Because of the development of new media technology, image production technology breakthrough and language communication ability, regardless from the graphics, video technology, or virtual reality digital expression, so that the visual information dissemination in the content and form of transformation, development has become a multi-faceted, full range of visual language.

Just as Cassirer said: "symbolic thinking and symbolic behavior is human life the most rich in representative features, and the full development of human culture is dependent on the conditions." Visual language form is one of the core forms of human civilization, which is based on the visual symbol system and constructed by the communication. As a kind of cultural form, the dominant position of the visual image occupies a key position in today 's communication and information dissemination, and it becomes more and more important in modern information communication. It not only changes the way people receive information, but also exerts a subtle influence on people's thinking mode and attitude.

The image of the times of the visual language form, by nature is the symbol of "figure" and the objective physical image of "like" the organic unity of, can not be separated with the symbol form and semantic dual attribute (the traditional visual culture studies to "ontology in symbolic form, object semantic symbol" mirror theory and formalism concept). Therefore, it not only has in the form of visual aesthetic feeling, but also has the ability of creation of meaning expression and artistic discourse. It is not naturally generated, but is constructed by the material of the symbolic language form, only in people's social environment and the exchange of communication in the state to be meaningful. The ordinary image without the purpose and intention of communication is not concerned in this paper.

As symbolic elements, from the form to the image, all of which have the nature of the simplified form and the typical characteristics of the decomposition of the morpheme, are "like" the basic category. The "map" is the premise of the 
symbolic "image", and it is the imitation, the record, the mapping and the reconstruction of the human's "image". It constructs a combination of multiple structural relations in the form of visual form and emotional function, and the grammatical function of its meaning, so that the image has a profound cultural and social significance.

Because of the intention of the image is always generated and spread in the man-made purpose, it is a specific visual element (morpheme) through the organic combination, so it can be formed in the specific context of the visual rhetoric and metaphor. It is different in pure reproduction imitation realism era of artistic skills, adopted the boundaries of time and space constraints of visual morpheme combination technique without, and timely use conditions for the development of science and technology of image making technology, the visual language of form produced amazing performance. This is also the fundamental reason for the rapid increase of the visual culture and visual culture today.

In the static visual representation of the creative, the movement of modern western painting is the beginning of the experiment in the visual arts, Cezanne, Van Gogh, has broken the pure realism and imitation modeling concept, deep into the combination, free form of visual reconstruction creation exploration; application in communication design field, especially the posters, commercial illustration etc. the expression form, different combinations of elements of "isomorphism" to build the image meaning of rhetoric, has become the basic mode and means of creative expression; and in the dynamic image field, the interpretation by the "Curitiba Schopf effect" of the "movie" montage, also is a typical language in more than two different lenses connecting produce meaning creation technique, the creation of such skills has become the basic grammar and structure of dynamic image work today. In addition, the creative performance of the digital technology, such as film and video games and other virtual reality, further enrich and deepen the visual language form and effect, and constantly boost the visual production technology and demand in the field of human visual creation, so that the effectiveness of visual language dissemination in the media or in the promotion of publishing.

The high quality vision which is supported by the modern science and technology, and the meaning combination and rhetoric of the visual elements, begin to produce the new visual logical discourse of the fantasy. Whether is cartoon, poster illustration, or film and television dramas, "Avatar" let we evoke the visually stunning visual performance in various fields have already become a reality. This is a distinction in the imitation and reappearance of the new image of the world, using graphics and visual production technology to achieve the visual model, simulation and quasi like, with "to lie said truth" concept and metaphorical and symbolic ability, with non realistic concept context reconstruction of reality.

This is a symbol of the language of the language of the amazing amazing language force and force. It constructs the external forms and emotional effects of beauty, but also pays more attention to the expression of personality, ideas and thoughts. At the same time, it further expands the dimension and depth of visual information expression. There is no doubt that the form of visual language, meaning construction and generation is the basis of visual culture and communication, but also the source and fundamental of visual art creation.

\section{REFERENCE}

[1] Bo Fei Ge, "The network media of visual communication design", master's degree thesis, Art Design department of Hunan University, 2002.

[2] Zhu Yongming, "The visual language form and communication strategy in the information age", the Chinese publishing, August 2010 . 